\title{
Extended van Hove Singularity and Superconducting Instability in Doped Graphene
}

\author{
J. L. McChesney, ${ }^{1,2}$ Aaron Bostwick, ${ }^{1}$ Taisuke Ohta, ${ }^{1,3}$ Thomas Seyller, ${ }^{4}$ Karsten Horn, ${ }^{3}$ J. González, ${ }^{5}$ and Eli Rotenberg ${ }^{1}$ \\ ${ }^{1}$ Advanced Light Source, Lawrence Berkeley National Laboratory, Berkeley, California, USA \\ ${ }^{2}$ Montana State University, Bozeman, Montana, USA \\ ${ }^{3}$ Fritz-Haber-Institut der Max-Planck-Gesellschaft, Berlin, Germany \\ ${ }^{4}$ Institut für Physik der Kondensierten Materie, Universität Erlangen-Nürnberg, Erlangen, Germany \\ ${ }^{5}$ Instituto de Estructura de la Materia, Consejo Superior de Investigaciones Científicas, Serrano 123, 28006 Madrid, Spain
}

(Received 18 November 2009; published 2 April 2010)

\begin{abstract}
We have investigated the effects of doping on a single layer of graphene using angle-resolved photoemission spectroscopy. We show that many-body interactions severely warp the Fermi surface, leading to an extended van Hove singularity (EVHS) at the graphene $M$ point. The ground state properties of graphene with such an EVHS are calculated, analyzing the competition between a magnetic instability and the tendency towards superconductivity. We find that the latter plays the dominant role as it is enhanced by the strong modulation of the interaction along the Fermi line, leading to an energy scale for the onset of the pairing instability as large as $1 \mathrm{meV}$ when the Fermi energy is sufficiently close to the EVHS.
\end{abstract}

DOI: 10.1103/PhysRevLett.104.136803

The fundamental interactions between charge, spin, and lattice depend very much on the topology of electronic bands, particularly in two dimensions when the Fermi level $E_{F}$ is near a saddle point. Such a saddle point occurs where the curvature of the bands has opposite sign in two orthogonal directions, leading to a van Hove singularity (VHS), i.e., a divergence in the density of states (DOS).

Much attention has been given to a "VHS scenario" in the cuprates, whereby the superconductivity is strongly influenced or even induced by a saddle point VHS [1,2]. Among the important effects of the VHS are (1) the presence of both electronlike and holelike carriers, leading to an attractive potential favoring pairing, (2) a high DOS, held to favor not only superconductivity but structural and magnetic instabilities, and (3) a perfect screening at wave vectors connecting VHSs, which acts to reduce repulsion. The topology of the VHS is important, especially when the curvature of one band vanishes; i.e., the electron or hole mass diverges. This increases the strength of the divergence in the DOS at such a so-called extended VHS (EVHS), which can increase the critical temperature for superconductivity [3].

Graphene's band structure has saddle points at the $M$ point of the Brillouin zone (BZ) occurring around $\pm 2 \mathrm{eV}$ from the charge neutrality point at the Dirac energy $E_{D}$ [4]. Since superconductivity occurs in graphene-related systems such as graphite-intercalation compounds and nanotubes, it is interesting to explore whether superconductivity or other instability can appear in graphene itself due to the influence of this VHS.

In this Letter, we present the band structure of highly doped graphene determined by angle-resolved photoemission spectroscopy (ARPES). We show that by chemically doping graphene on both sides, a much higher level of
PACS numbers: 73.22.Pr, 73.20.At, 74.70.Wz, 79.60.-i

doping can be achieved than previously obtained. By this method, we can induce an electronic topological transition in graphene for the first time, whereby the Fermi energy $E_{F}$ is brought to the position of the saddle point VHS in graphene. We find that the electronic structure is strongly renormalized by the resulting DOS divergence such that the VHS has an extended, not pointlike, character [5,6]; feeding the experimental Fermi surface topology into a calculation of the screened Coulomb interaction, we predict the system is unstable to superconducting pairing due to electron-electron interactions.

Clean graphene on $\mathrm{SiC}(0001)$ was prepared as before $[7,8]$, and various combinations of $\mathrm{K}$ and $\mathrm{Ca}$ were used as dopants. Ca could be intercalated under the graphene by repeated cycles of $\mathrm{Ca}$ deposition and annealing onto slightly incomplete graphene layers, which consist of isolated, but nearly merged islands of monolayer graphene. Once intercalated underneath, the $\mathrm{Ca}$ atoms were stable up to the $\mathrm{SiC}$ decomposition temperature; further doping could be achieved by adsorbing additional $\mathrm{Ca}$ or $\mathrm{K}$ atoms on top of the graphene. Potassium was prepared in the dispersed, disordered phase [9], or else the ordered $\mathrm{K}(2 \times$ 2) phase by depositing at low $(20 \mathrm{~K})$ or high $(100 \mathrm{~K})$ temperature, respectively. Unlike $\mathrm{K}, \mathrm{Ca}$ atoms showed no long-range order (expected to be of $\sqrt{3} \times \sqrt{3}-R 30^{\circ}$ symmetry as in $\mathrm{CaC}_{6}$ ) which would lead an energy gap at $E_{D}$ [10] and satellite bands.

Figures 1(a)-1(h) show a series of Fermi surfaces and underlying energy bands as a function of doping. The conical Dirac-like electron pockets centered at the corners $K, K^{\prime}$ of the hexagonal $\mathrm{BZ}$ are all characterized by kinks $\sim 200 \mathrm{meV}$ below $E_{F}$ due to $e$-ph coupling and kinks at the Dirac crossing energy $E_{D}$ due to electron-plasmon coupling as reported previously [8]; here the data are presented for significantly more doping. 


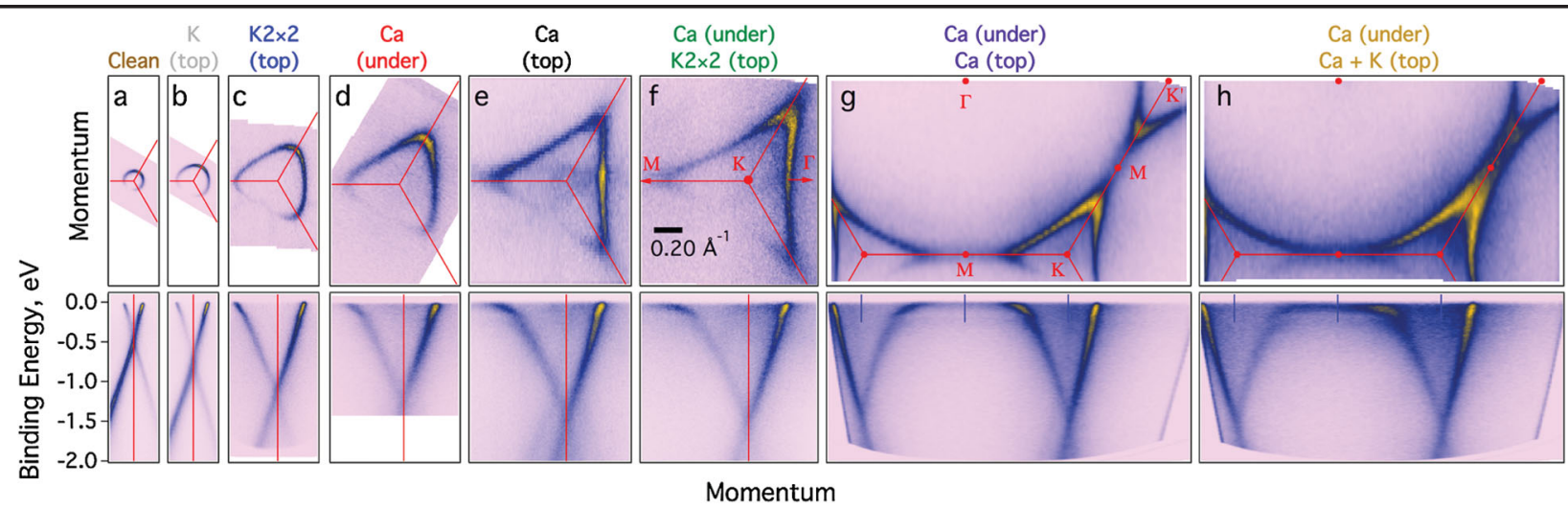

FIG. 1 (color). Doping dependence of (upper) Fermi surface and (lower) band structure of $\pi$ bands along the $M K \Gamma$ directions, for various combinations of doping from (a) as-grown graphene, (b)-(h) graphene with $\mathrm{Ca}$ and/or K deposition as labeled. The scale bars in (f) apply to parts (a)-(f) only.

With the last film [Fig. 1(h)], we achieved a doping just higher than the VHS level. Now the triangular electron pockets at $K, K^{\prime}$ have merged and transformed into a large hole pocket centered at $\Gamma$. This is demonstrated in Fig. 2, which shows the energy bands along and perpendicular to the $K-K^{\prime}$ line of the graphene BZ. Along this line, the bands no longer cross $E_{F}$ as in Figs. 1(a)-1(g), and across it we can resolve the shallow parabolic band characterizing the hole states above the VHS.

The most notable feature of Figs. 1 and 2 is the high degree of trigonal warping just below the VHS, and the corresponding flatness of the band along the VHS. The lack of energy dispersion in one direction demonstrates an extended, not pointlike, VHS. Such an EVHS cannot be reconciled with existing calculations, as shown by comparing the measured band along the EVHS to three calculations: an elementary near-neighbor (NN) tight-binding (TB) model [4], and third-NN TB fits to local-density approximation (LDA) [11] and $G W$ calculations [12]. Not only is the experimental band much flatter than in these models, but also the VHS at the $M$ point occurs at much lower energy. This is true even considering a $7 \%$ reduction in bandwidth predicted for our highest doping [13]. In contrast, the bands observed near the saddle point below $E_{D}$ (not shown) show a quite ordinary pointlike VHS resembling Fig. 3(a).

A combined plot of the Fermi surfaces in Fig. 3(e) shows that they evolve smoothly with doping, independent of the particular combination of dopant atom. In particular, the samples in Figs. 1(f) and 1(g) have indistinguishable Fermi surfaces despite their different dopant composition. This insensitivity to the dopant type suggests that hybridization between $\pi^{*}$ and $\mathrm{Ca}$ states does not enhance the trigonal warping of the bands as proposed in Ref. [10] as would be expected by the lack of $\sqrt{3} \times \sqrt{3}-R 30^{\circ}$ symmetry in our samples.

We have fitted the experimental bands to an empirical third-NN TB model $[11,14]$, plotted in Fig. 2. Our model is not perfect: first, it misses the notable kinks at $E_{F}-200 \mathrm{meV}$ due to mass renormalization from $e$-ph cou- pling [8,15-17]. These kinks further flatten the bands and extend the length of the EVHS. Second, the model band is not perfectly flat at $M$. But the broadness of the data admits the possibility of some finite curvature at $M$, so the model represents a conservative representation of the EVHS, ignoring its enhancement by $e$-ph interactions. A comparison of the constant-energy surfaces of all the models (after scaling to match the measured VHS energy) with the experimental Fermi surfaces, see Fig. 3, shows that even after scaling the models to the experimental bandwidth, our empirical model best represents the length and breadth of the EVHS, with $G W$ the next closest.

We believe that the EVHS arises due to the renormalization of the dispersion, self-induced by its own diverging DOS by its proximity to $E_{F}$. Such effects were predicted generally for 2D systems [5] and for graphene [6]. This is

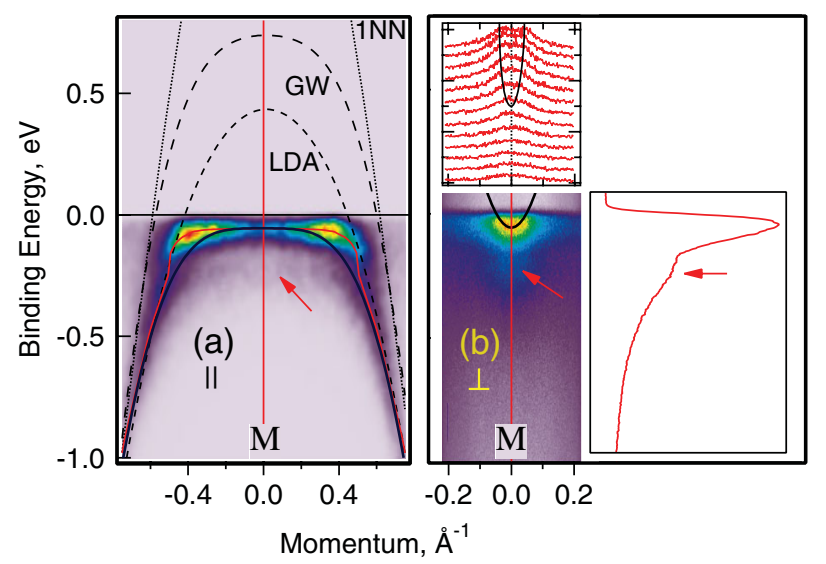

FIG. 2 (color). The spectral function for the sample in Fig. 1(h) (a) along and (b) across the $K-K^{\prime}$ line through the $M$ point. The dotted black lines and the dashed black lines are model energy bands from the literature $[4,11,12]$. The full line is an empirical tight-binding model from this work. The red solid line is a guide to the measured energy band. The red arrows indicate the incoherent mode arising from phonon coupling as described in the text. The insets to (b) are, above, momentum distribution curves across the $M$ point as a function of binding energy, and, right, angle-integrated energy distribution at $M$. 




FIG. 3 (color). (a)-(d) Tight-binding fits to various published graphene model band structures [4,11,12] and our empirical band structure [14]. The gray regions highlight the waist of the VHS, defined as the range where the width varies by $<5 \%$. (e) The experimental Fermi surfaces for the samples in Figs. 1(a) -1 (h), overlaid on an image of the constant-energy surface at the VHS from the sample in Fig. 1(h) $\left(55 \mathrm{meV}\right.$ below $\left.E_{F}\right)$ [34].

also supported by the more favorable agreement of the $G W$ model, which partially accounts for the $e$-e interaction.

The $e$-ph interaction adds an additional mass term near the EVHS, further enhancing its length and the DOS singularity. (The flattening of the saddle point dispersion induced by the divergent DOS is a second-order effect in the interaction potential [5], so that it can receive added contributions from both the Coulomb and the phononmediated interactions.) Therefore the properties that follow from the EVHS arise from both electron-electron $(e-e)$ and $e$-ph interactions, which are themselves modified or enhanced by the EVHS.

The importance of the $e$-ph coupling is suggested by the observed spectral weight transfer from the quasiparticle near $E_{F}$ to a "hump" $200 \mathrm{meV}$ below the EVHS (not $E_{F}$ ), whose intensity is larger than can be accounted for in a simple $k$-independent $e$-ph coupling model. This hump may arise from polaron formation [18] since near the EVHS the system is in an "anti-Born-Oppenheimer" limit: the band velocity is zero so that electronic motion is slower than lattice motion. Therefore as the photohole hops from $\mathrm{C}$ to $\mathrm{C}$, the lattice has time to rearrange; the hump represents incoherent intensity resulting from the dressing of the quasiparticles by phonons [19].

Regardless of the detailed nature of the many-body interactions underlying the extended VHS, it is known that the divergent DOS leads to an instability in the electron system. A possible ferromagnetic ground state has been demonstrated when the Fermi line degenerates as in Fig. 3(d) [20]. Moreover, the strong modulation of the Fermi velocity along the Fermi line and its effect on the interaction vertex may give rise to a pairing instability, following the mechanism proposed by Kohn and Luttinger [21]. Previous analyses of the electron system near a VHS have shown that the screening by the singular DOS at the VHS is sufficiently strong that only the local Coulomb interaction $U$ is important at low energies $[5,22]$. Then, the interaction vertex for the BCS kinematics $V\left(\mathbf{k}, \mathbf{k}^{\prime}\right)$ is dressed in the form [23]

$$
V\left(\mathbf{k}, \mathbf{k}^{\prime}\right)=\frac{U}{1-U \chi_{0}\left(\mathbf{k}+\mathbf{k}^{\prime}\right)}+\frac{U^{3} \chi_{0}^{2}\left(\mathbf{k}-\mathbf{k}^{\prime}\right)}{1-U^{2} \chi_{0}^{2}\left(\mathbf{k}-\mathbf{k}^{\prime}\right)},
$$

where $\chi_{0}(\mathbf{q})$ is the particle-hole susceptibility. Thus, even when the interaction $U$ is repulsive, the strong modulation of $\chi_{0}(\mathbf{q})$ along the Fermi line may lead to an attractive coupling.

We have computed $\chi_{0}(\mathbf{q})$ in lattices with up to $800^{2}$ points in order to evaluate the BCS vertex $V\left(\mathbf{k}, \mathbf{k}^{\prime}\right)$ and ultimately the effective coupling parameters $\lambda^{(\gamma)}$, where the vertex is projected into the different irreducible representations $\gamma$ of the hexagonal point symmetry group. These are very sensitive to the on site interaction $U$. The most remarkable feature is that, regardless of the sign of the interaction $U$ (repulsive or attractive), there is always a dominant attractive coupling $\lambda^{(\gamma)}$ as a consequence of the EVHS, driving the system to a superconducting instability when the Fermi level is placed close enough to the EVHS.

Taking the value of $U=2.1 \mathrm{eV}$ from graphite [24], we find the dominant coupling constant $\lambda^{(\gamma)} \approx-0.12$ in the representation $\gamma$ given by the $\cos (3 \theta)$ basis function, for a deviation of the Fermi level $\mu=1 \mathrm{meV}$ with respect to the VHS. This can be compared to the case of the ordinary VHS that occurs at the $M$ point $\sim 2.5 \mathrm{eV}$ below $E_{D}$, where we find $\lambda^{(\gamma)} \approx-0.075$. If we consider that the critical scale of the pairing instability is proportional to $e^{-1 /\left|\lambda^{(\gamma)}\right|}$, such a variation of the coupling translates into a difference of 2 orders of magnitude in the critical temperatures expected in the two cases.

Figure 4 shows the calculated dominant coupling constant $\lambda^{(\gamma)}$ as contours in the space of $U$ and $\mu$. We find that the system is unstable to superconductivity throughout the range studied. In addition, we have also established the phase diagram for magnetism by computing the dependence on temperature $T$ of the uniform susceptibility $\chi(\mathbf{0})=\chi_{0}(\mathbf{0}) /\left[1-U \chi_{0}(\mathbf{0})\right]$. We find that, at sufficiently low $T, \chi(\mathbf{0})$ always has a divergence marking an upper bound for the onset of a magnetic instability, which is represented in Fig. 4 for two different transition temperatures. It is clear, however, that for $U \sim 2 \mathrm{eV}$ and above, the values of the dominant coupling $\lambda^{(\gamma)}$ are so large that the superconducting instability will always appear first at a higher temperature.

It is tempting to relate these results to the superconductivity of the graphite-intercalation compounds, most notably $\mathrm{CaC}_{6}$, which has the highest value of $T_{c}=11.6 \mathrm{~K}$ [25]. Despite an early attribution to $e-e$ interaction [26], the superconductivity has been well understood in terms of $e$-ph coupling [27-30], although some unresolved issues remain [30].

Neither ARPES measurements [31,32] nor theoretical calculations of the band structure [27-30] and DOS [33] indicate a strong singularity in $\mathrm{CaC}_{6}$ at $E_{F}$ expected for an EVHS. Part of the reason is that in $\mathrm{CaC}_{6}$ the neighboring $\mathrm{Ca}$ interlayers have different stacking, so that the doped graphene band - originally near the VHS - is split into two bands just above and just below the VHS. In addition, the folding of bands from different parts of the graphene BZ due to the rhombohedral symmetry mixes the $\mathrm{Ca}$ and 


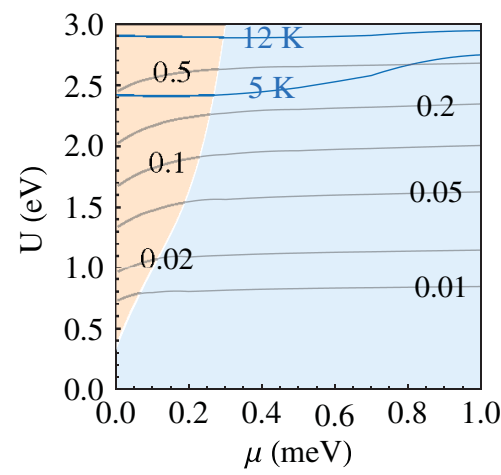

FIG. 4 (color). Phase diagram showing the contours of constant coupling $|\lambda|$ (black lines) and constant transition temperature for the ferromagnetic instability (blue lines) as a function of the local Coulomb interaction $U$ and the Fermi energy $\mu$ relative to the VHS. The two colored regions correspond to different order parameters of the pairing instability with the symmetry of the $\cos (3 \theta)$ representation (blue) and that of the $\{\cos (2 \theta), \sin (2 \theta)\}$ representation (orange) of the point group.

C states, further disrupting the band structure [27]. So at present there is no reason to believe that electron-electron interactions as described here play a dominant role in $\mathrm{CaC}_{6}$ superconductivity.

That said, our data strongly support the idea that doped graphene can achieve an electronically mediated superconductivity provided that the doping is in the vicinity of the VHS and the lattice symmetry is preserved; i.e., the chemical dopants do not introduce new states near the VHS to disrupt the band structure.

We are grateful to R. Markiewicz and C. Y. Kim for discussions. Research at the Advanced Light Source was supported by the Director, Office of Science, Office of Basic Energy Sciences, of the U.S. Department of Energy. J. G. acknowledges the financial support of MICINN (Spain) through Grant No. FIS2008-00124/FIS.

[1] D. M. Newns, H. R. Krishnamurthy, P.C. Pattnaik, C.C. Tsuei, and C. L. Kane, Phys. Rev. Lett. 69, 1264 (1992).

[2] R. S. Markiewicz, J. Phys. Chem. Solids 58, 1179 (1997).

[3] K. Gofron, J.C. Campuzano, A.A. Abrikosov, M. Lindroos, A. Bansil, H. Ding, D. Koelling, and B. Dabrowski, Phys. Rev. Lett. 73, 3302 (1994).

[4] R. Saito, G. Dresselhaus, and M. S. Dresselhaus, Physical Properties of Carbon Nanotubes (Imperial College Press, London, 1998).

[5] J. González, F. Guinea, and M. A. H. Vozmediano, Nucl. Phys. B485, 694 (1997).

[6] R. Roldán, M. P. López-Sancho, and F. Guinea, Phys. Rev. B 77, 115410 (2008).

[7] I. Forbeaux, J. M. Themlin, and J. M. Debever, Phys. Rev. B 58, 16396 (1998).

[8] A. Bostwick, T. Ohta, T. Seyller, K. Horn, and E. Rotenberg, Nature Phys. 3, 36 (2007).
[9] P. Bennich, C. Puglia, P. A. Brühwiler, A. Nilsson, A. J. Maxwell, A. Sandell, N. Martensson, and P. Rudolf, Phys. Rev. B 59, 8292 (1999).

[10] M. Calandra and F. Mauri, Phys. Rev. B 76, 161406(R) (2007).

[11] S. Reich, J. Maultzsch, C. Thomsen, and P. Ordejón, Phys. Rev. B 66, 035412 (2002).

[12] A. Gruneis, C. Attaccalite, L. Wirtz, H. Shiozawa, R. Saito, T. Pichler, and A. Rubio, Phys. Rev. B 78, 205425 (2008).

[13] C. Attaccalite, A. Gruneis, T. Pichler, and A. Rubio, arXiv:0808.0786.

[14] The empirical model uses (1st, 2nd, 3rd) NN hopping parameters $(-3.02,-1,-.46) \mathrm{eV}$ and 1 st $\mathrm{NN}$ overlap $0.2 \mathrm{eV}$.

[15] M. Calandra and F. Mauri, Phys. Rev. B 76, 205411 (2007).

[16] W.-K. Tse and S. Das Sarma, Phys. Rev. Lett. 99, 236802 (2007).

[17] C.-H. Park, F. Giustino, M. L. Cohen, and S. G. Louie, Phys. Rev. Lett. 99, 086804 (2007).

[18] R. S. Markiewicz, J. Phys. Chem. Solids 56, 1637 (1995).

[19] L. Perfetti, H. Berger, A. Reginelli, L. Degiorgi, H. Höchst, J. Voit, G. Margaritondo, and M. Grioni, Phys. Rev. Lett. 87, 216404 (2001).

[20] R. Hlubina, S. Sorella, and F. Guinea, Phys. Rev. Lett. 78, 1343 (1997).

[21] W. Kohn and J. M. Luttinger, Phys. Rev. Lett. 15, 524 (1965).

[22] J. González, Phys. Rev. B 67, 054510 (2003).

[23] D. J. Scalapino, E. Loh, and J. E. Hirsch, Phys. Rev. B 35, 6694 (1987).

[24] E. Perfetto, M. Cini, S. Ugenti, P. Castrucci, M. Scarselli, M. De Crescenzi, F. Rosei, and M. A. El Khakani, Phys. Rev. B 76, 233408 (2007).

[25] T.E. Weller, M. Ellerby, S. S. Saxena, R. P. Smith, and N. T. Skipper, Nature Phys. 1, 39 (2005).

[26] G. Csanyi, P. B. Littlewood, A. H. Nevidomskyy, C. J. Pickard, and B. D. Simons, Nature Phys. 1, 42 (2005).

[27] M. Calandra and F. Mauri, Phys. Rev. Lett. 95, 237002 (2005).

[28] J. S. Kim, L. Boeri, R. K. Kremer, and F. S. Razavi, Phys. Rev. B 74, 214513 (2006).

[29] A. Sanna, G. Profeta, A. Floris, A. Marini, E. K. U. Gross, and S. Massidda, Phys. Rev. B 75, 020511(R) (2007).

[30] I. I. Mazin, L. Boeri, O. V. Dolgov, A. A. Golubov, G. B. Bachelet, M. Giantomassi, and O. K. Andersen, Physica (Amsterdam) 460-462C, 116 (2007).

[31] T. Valla, J. Camacho, Z.H. Pan, A. V. Fedorov, A. C. Walters, C. A. Howard, and M. Ellerby, Phys. Rev. Lett. 102, 107007 (2009).

[32] K. Sugawara, T. Sato, and T. Takahashi, Nature Phys. 5, 40 (2009).

[33] S. Q. Deng, A. Simon, and J. Kohler, Angew. Chem. 47, 6703 (2008).

[34] The image data is extracted from the data set of Fig. 1(h), with the missing parts filled in using the mirror-plane symmetries, and with a simple linear scaling factor in the $y$ direction to restore mirror symmetry in the intensity. 